\title{
Direct Conversion of Human Preadipocytes into Hematopoietic, Neuronal, and Pancreatic $\alpha$ Cells by Oct4 and KIf4 Overexpression
}

\author{
Jong Seop Rim, ${ }^{1, *}$ Phillip Lewis, ${ }^{1}$ Atman Dave, ${ }^{1}$ Juyoung Rim, ${ }^{1}$ Kimberly Szuszka, ${ }^{1}$ Cristina Acosta, ${ }^{1}$ Joohyun Kim, ${ }^{2}$ \\ Jeong-Do Kim, ${ }^{1}$ Jaroslaw Staszkiewicz, ${ }^{1}$ Ru Gao, ${ }^{1}$ Lettie Harkins, ${ }^{1}$ and Kenneth J. Eilertsen ${ }^{1,3, *}$
}

\begin{abstract}
Cellular plasticity obtained by natural adaptation or by forced expression of developmental stage and lineagespecific transcription factors converts differentiated cells into three different levels of potency: pluripotent, multipotent (dedifferentiation), and oligo- or unipotent (transdifferentiation). These processes hold promise for regenerative medicine to replace damaged cells and organs through the identification of transcription factors that determine the fate of cellular plasticity. In this study, we report that ectopic expression of Oct4 and Klf4 induces transdifferentiation of human preadipocytes into three different lineages: hematopoietic, neuronal, and pancreatic endocrine cells. Furthermore, ectopic expression of Oct4 is sufficient to induce transdifferentiation of preadipocytes into glucagon-expressing pancreatic $\alpha$ cells. This will provide a simple and efficient method to produce functionally competent cells for therapeutic regenerative medicine.
\end{abstract}

Key words: developmental biology; gene expression; molecular biology; regeneration; stem cells

\section{Introduction}

Overexpression of defined transcription factors can convert one cell type into another and has important implications for regenerative medicine. Ectopic expression of key transcription factors in somatic donor cells has been used to generate many different cell types, including cells resembling blood cells, ${ }^{1,2}$ brown fat cells, ${ }^{3}$ hepatocytes, ${ }^{4}$ sertoli cells, ${ }^{5}$ and various cell types of the neural lineage. ${ }^{6-9}$

Expression of Oct4, partially in conjunction with Klf4, is critical to maintain pluripotency at early developmental stages, including in the blastomere, inner cell mass (ICM), and epiblast. Oct 4 and Klf4 have been reported as components of four defined transcription factors (Oct4, Sox2, $\mathrm{Klf4}$, and $\mathrm{cMyc}$ ) that trigger reprogramming of somatic cells into pluripotent stem cells (induced pluripotent stem [iPS] cell) that are functionally identical to embryonic stem cells. ${ }^{10}$ Ectopic expression of Oct4 alone, or ectopic expression of Oct4 and Klf4, in combination with small molecule treatment, is sufficient to generate iPS cells from neural stem cells, mouse embryonic fibroblasts (MEFs), and dermal papilla cells. ${ }^{11-14}$ Oct4 activates early epithelial-to-mesenchymal transition (EMT), which is critical to somatic cell reprogramming. ${ }^{15}$ Interestingly, Szabo et al. demonstrate direct conversion of human dermal fibroblasts into multipotent blood progenitors by ectopic expression of Oct4. ${ }^{16}$ This suggests that Oct4 functions as a hematopoietic lineage differentiation marker as well. In this study, we report that Oct4 and Klf4 induce transdifferentiation of human preadipocytes into three different lineages, including hematopoietic, neuronal, and pancreatic endocrine cells. Furthermore,

\footnotetext{
${ }^{1}$ NuPotential, Inc., Baton Rouge, Louisiana.

${ }^{2}$ Center for Computation and Technology, Louisiana State University, Baton Rouge, Louisiana.

${ }^{3}$ Pennington Biomedical Research Center, LSU System, Baton Rouge, Louisiana.
}

*Address correspondence to: Jong Seop Rim, DVM, PhD, NuPotential, Inc., Louisiana Emerging Technology Center, 340 East Parker Blvd, Baton Rouge, LA 70803, E-mail: jong@nupotential.com or Kenneth J. Eilertsen, PhD, Pennington Biomedical Research Center, LSU System, 6400 Perkins Road, Baton Rouge, LA 70808, Email: Kenneth.Eilertsen@pbrc.edu

(c) Jong Seop Rim et al. 2015; Published by Mary Ann Liebert, Inc. This Open Access article is distributed under the terms of the Creative Commons License (http://creativecommons.org/licenses/by/4.0), which permits unrestricted use, distribution, and reproduction in any medium, provided the original work is properly credited. 
ectopic expression of Oct 4 alone is sufficient to induce transdifferentiation of preadipocytes into glucagonexpressing pancreatic $\alpha$ cells.

\section{Results and Discussion}

Ectopic expression of Oct4 and Klf4 induces transdifferentiation of human preadipocytes

Primary cultures of preadipocytes from stromal vascular fraction of adipose tissues are known as a rich source of CD34 and alkaline phosphatase-positive adult mesenchymal stem cells. ${ }^{17}$ Preadipocytes are capable of differentiating toward mesodermal lineages, including osteocyte, adipocyte, and chondrocyte (Supplementary Fig. S1a). We generated two-factor (OK) and four-factor (OKSM) transfected cells using highly proliferative, low passage preadipocytes ( $<3$ passages) that had been demonstrated to have $\sim 50 \%$ transfection yields using a lentiviral vector (Supplementary Fig. S1b). Our general strategy is outlined in Figure 1a. One day before lentiviral infection, cells were seeded on a six-well plate at the concentration of $10^{5}$ cells per well with Dulbecco's Modified Eagle's Medium (DMEM) containing $10 \%$ fetal bovine serum (FBS). For the lentiviral infection, total 25 multiplicity of infection (MOI) of lentivirus that expresses Oct4, Klf4 under control of cytomegalovirus promoter (SBI and Cellomics), or polycistronic lentivirus STEMCCA that expresses four iPS factors (Oct4, Sox2, Klf4, and cMyc) were added to the medium with $5 \mu \mathrm{g} / \mathrm{mL}$ polybrene (Sigma-Aldrich) (Fig. 1a). The lentiviral infection was repeated the next day, and the culture medium was changed with fresh DMEM/5\% FBS medium every other day. When cells became confluent, they were trypsinized, counted, and seeded on MEF feeder layer at a density of $5 \times 10^{4}$ cells $/ \mathrm{mL}$ with the mTeSR 1 medium (StemCell Technology). Emerging of round cells with colony formation was observed as early as 5 days post feeder layer cultures. The two-factor (OK)- transfected cells showed similar morphological changes when compared to the four iPS factor (OSKM)-transfected cells that were undergoing early stages of somatic cell reprogramming (Fig. 1a), with the exception that more dense colony formation and immortalization from the OSKM-overexpressed iPS colonies (adiposederived iPS cell [AdiPS cells]) were observed. Interestingly, similar morphological changes have been shown from multilineage blood progenitors through overexpression of Oct4 in human adult dermal and neonatal foreskin fibroblasts. ${ }^{16}$ We established nine OK cell lines and multiple iPS cell lines from OSKM-overexpressed preadipocytes by colony picking.
To further characterize OK-transfected cells, we examined global gene expression patterns using microarray analysis (Illumina). Sample relations based on the expression of 20,909 genes showed that OK cells (single clone, mixed colonies) are distinct from preadipocyte and four-factor (OSKM)-induced iPS cells (AdiPS) (Fig. 1b). A majority of the pluripotency genes that were highly expressed in the fully reprogrammed AdiPS cells were expressed at lower levels in two-factor-induced $\mathrm{OK}$ cells, but at levels similar to or less than levels observed in preadipocytes (Fig. 1c). Expression of fibroblast and preadipocyte marker genes was expressed at lower levels in OK cells with the exception of EMT activators (Slug, $\mathrm{N}$-Cad), which maintained higher expression levels (Fig. 1d). In contrast to a previous report, ${ }^{15}$ this suggested that OK cells underwent an EMT rather than reprogramming processes. Epigenetic modifiers, including inhibitors for histone deacetylase (HDAC), histone methyltransferase (HMT), and DNA methyltransferase (DNMT), are known to facilitate the reprogramming process by targeting epigenetic barriers. ${ }^{18-20}$ Small molecule combination treatments (e.g., BIX01294, BayK8644, RG-108) have been reported to enable two-factor (Oct4 and Klf4)-induced reprogramming of MEFs. ${ }^{18}$ In our hands, exposure to the DNMT1 inhibitor zebularine did not affect the reprogramming or global gene expression patterns (data not shown).

\section{Transdifferentiation of hematopoietic and neuronal lineages by Oct4 and Klf4 overexpression}

Using a BioGPS gene annotation portal (www.biogps .org), we analyzed the lineage and tissue-specific expression profiles of the top 292 genes characterized by a minimum fourfold change induced by Oct 4 and Klf4 overexpression. As summarized in Figure 2a, we observed the enrichments of specific genes from three germ layers (mesoderm, ectoderm, and endoderm) and ICM. Overall, $41 \%$ of selected genes are annotated as tissue-specific expression while the remaining is ubiquitously expressed. Specifically, a total of 45 genes are designated hematopoietic cell specific, 59 genes are brain and peripheral nerve specific, and 27 genes are exclusively expressed from pancreas and pancreatic islets. Oct4 is also known as a hematopoietic transcription factor that enhances the expression of pan-leukocyte marker CD45 in human fibroblasts. ${ }^{16}$ In the article, Oct4-derived fibroblasts $\left(\mathrm{CD} 45^{+} \mathrm{Fib}^{\mathrm{Oct} 4}\right)$ differentiate into mature blood cells by additional cytokine treatments. Unlike the Oct4-derived fibroblasts ( $\mathrm{Fib}^{\mathrm{Oct} 4}, \mathrm{CD} 45^{+} \mathrm{Fib}^{\mathrm{Oct} 4}$ ), OK cells do not alter the expression of hematopoietic transcription factors and 

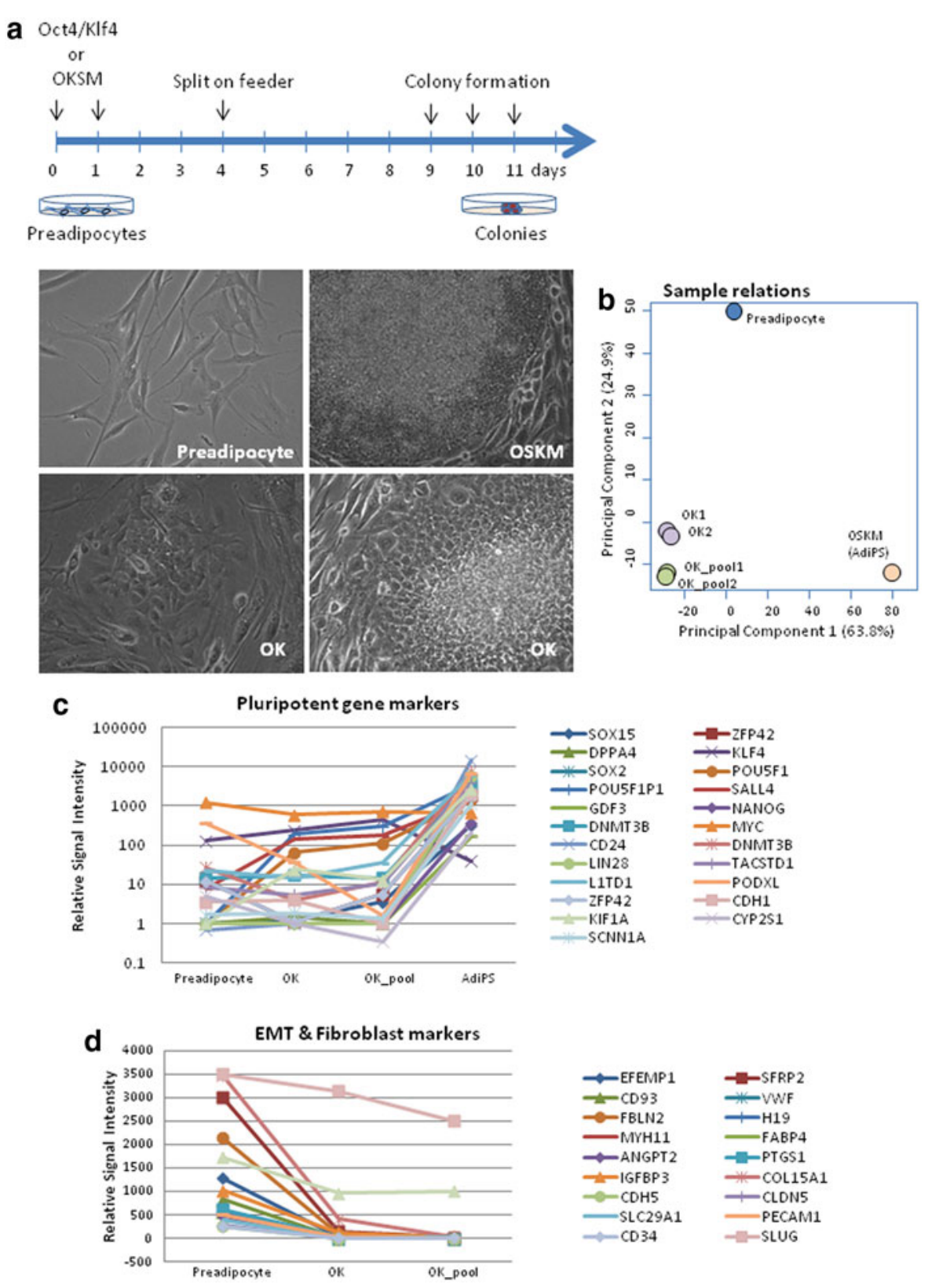

FIG. 1. Ectopic expression of Oct4 and Klf4 in human preadipocytes. (a) Experimental strategy and micrographs of colony formation during transdifferentiation and reprogramming of human preadipocyte by lentiviral overexpression. Lentivirus that overexpresses two factor (Oct4, KIf4) or four iPS factor (OSKM) was infected into human preadipocytes. Cells were maintained on MEF cell supplemented with the mTeSR1 medium. (b) Principal component analysis on gene expression data of microarray displays distinct cell populations (preadipocyte, OK; single clone, OK_pool; mixed colonies, AdiPS; iPS cell) and clustering of replicates $(n=2)$. (c) Relative signal intensity from microarray displays no significant changes in pluripotent markers that highly expressed in the fully reprogrammed AdiPS cells. (d) Relative signal intensity from microarray displays downregulation of fibroblast markers, while the expression of EMT activators (Slug, N-Cad) maintains highly. AdiPS, Adipose-derived iPS cell; EMT, epithelial-to-mesenchymal transition; iPS cell, induced pluripotent stem cell; MEF, mouse embryonic fibroblast. 

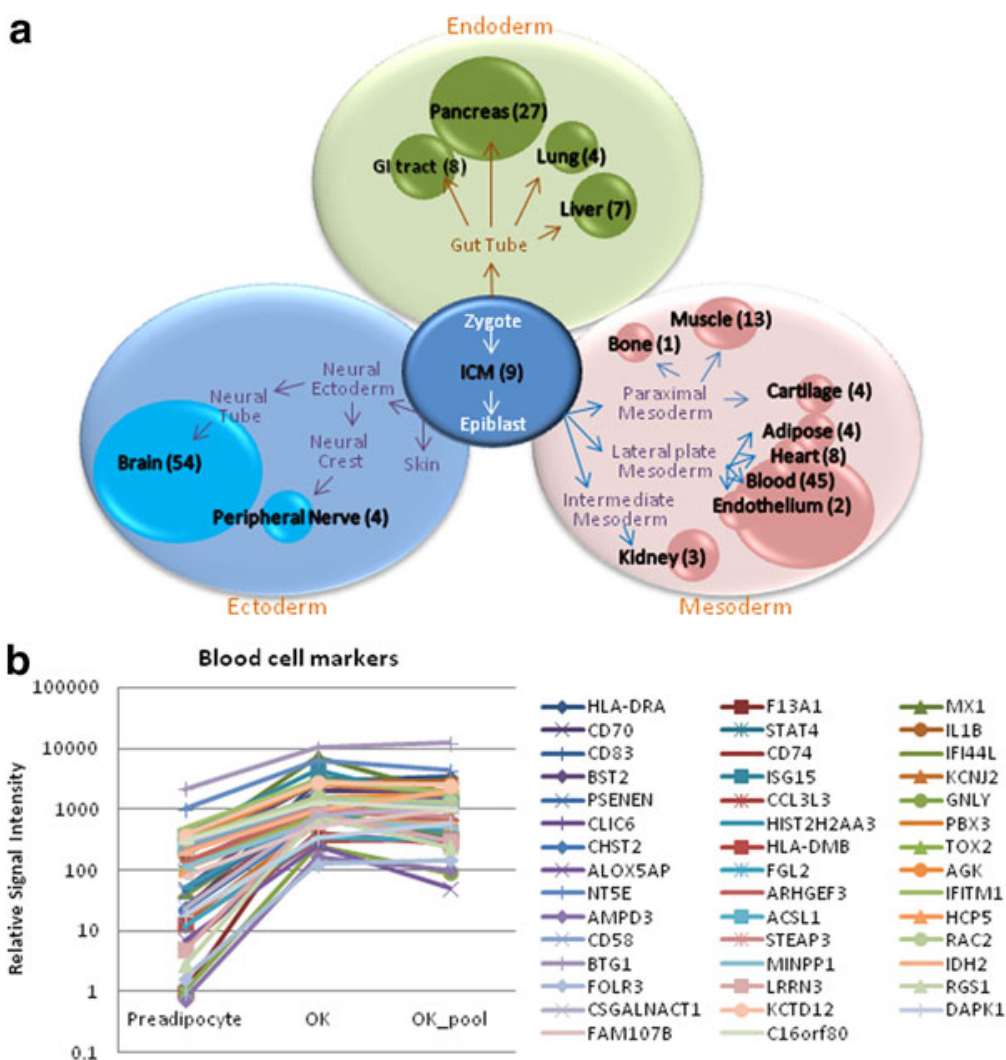

C
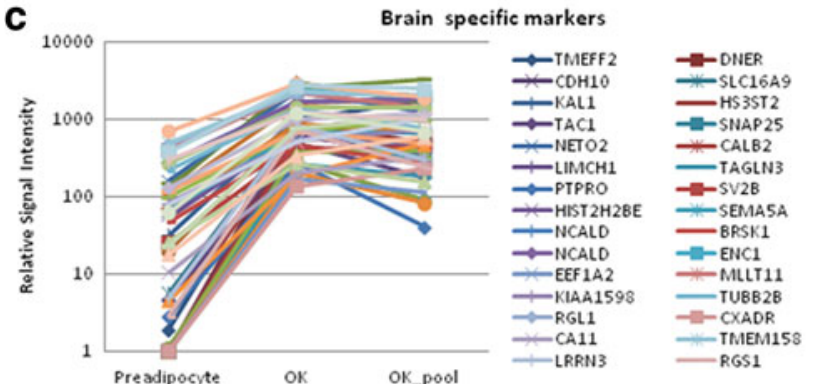

$$
\begin{aligned}
& \text { =IF16 } \\
& \text {-TPO52L1 }
\end{aligned}
$$
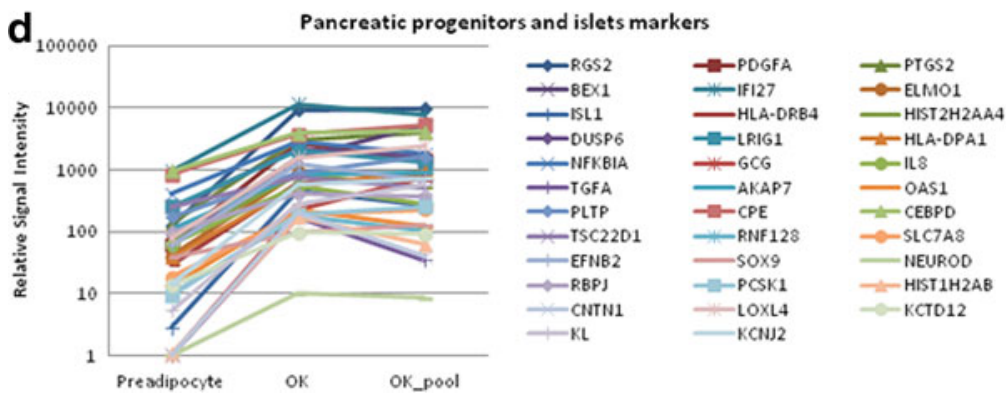

FIG. 2. Transdifferentiation of hematopoietic, neuronal, and pancreatic lineages by Oct4 and Klf4 overexpression. (a) BioGPS gene annotation. The number of lineage and tissue-specific genes is summarized. (b) Relative signal intensity from microarray displays upregulation of blood cell-specific gene expression from two-factor-induced OK cell (Single clone) and OK_pool (mixed colonies). (c) Brain-specific gene expression. (d) Pancreatic progenitors and islet-specific gene expression. 
cytokines (Supplementary Fig. S2). However, OK cells showed upregulation of $\mathrm{CD} 71^{+}$, early erythroid marker genes (CHST2, AMPD3, CD58, SREAP3, MINPP1, IDH2), lymphoblast marker genes (CD70, HLA-DRA, MX1, CD83, CD74, IFI44L, BST2, ISG15, CLIC6, PBX3, TOX2, HCP5, RAC2, RGS1), lymphocyte marker genes (STAT4, HLA-DMB, NT5E, HCP5, RAC2, BTG1), and $\mathrm{CD}^{+} 4^{+}$monocyte/dendritic cell marker genes (F13A1, FGL2, FOLR3, MX1, PSENEN, RGS1) (Fig. 2b).

Direct conversion of mouse fibroblasts into neurons by the combinatorial expression of neural lineage-specific transcription factors (Ascl1, Brn2, Myt1l) has been demonstrated previously. ${ }^{6}$ In addition, four reprogramming factor (Oct4, Sox2, Klf4, cMyc)-induced intermediate cells have been identified as neural progenitor cells. ${ }^{21}$ Interestingly, the tissue-specific gene expression pattern analysis revealed that $\mathrm{OK}$ cells expressed increased levels of brain-specific markers (IFI6, HS3ST2, TAGLN3, SV2B, NCALD, ENC1, COBL, EEF1A2, KBTBD11, RGL1, CA11). Some genes are known to be exclusively expressed from fetal brain (NETO2, DOK5, TUBB2B, PCDH19, PTPRO, SATB2, FGF13, CXADR, LPPR4, MLLT11) or regions specific to the amygdala (TMEFF2, BRSK1, NRGN, TAC1PCDH19), pineal glands (CDH10, TPD52L1, HIST2H2BE, NCALD, CXADR), prefrontal and cortex (SLC16A9, NRGN, MEGF10, FMNL2, EEF1A2, KIAA1598, KBTBD11, RGL1, CA11, SATB2), hypothalamus and thalamus (DNER, CALB2, FMNL2, KIAA1598), and spinal cord (KAL1, DNER, MEGF10). Most of the brain-specific genes that were highly induced in OK cells were undetectable or at very low levels when compared to untransfected human preadipocytes (Fig. 2c). Neuronal differentiation, however, did not proceed any further: OK cells did not show neuronal morphological changes such as neuronal rosettes or nerve fibers (axon) (data not shown). In addition, the expression of neural lineage-specific transcription factors (Ascl1, Brn2, Myt1l) were not altered in OK cells. These results suggest that Oct4- and Klf4-induced neural transdifferentiation is independent of neural development, and that Oct4 and Klf4 overexpression directly converts mesoderm to ectoderm lineages.

Transdifferentiation of pancreatic $\alpha$ cells by Oct4/Klf4 or Oct4 alone

As a part of an endocrine organ, pancreatic $\alpha$ and $\beta$ cells play vital roles maintaining blood glucose homeostasis by secreting glucagon and insulin, respectively. Three pancreatic lineage-specific transdifferentiation factors (Pdx1, Ngn3, Mafa) have been identified, which effi- ciently convert adult hepatocytes and pancreatic exocrine cells into insulin secreting pancreatic $\beta$ cells. $^{22-25}$ However, pancreatic $\alpha$ cell-specific transdifferentiation has not been reported. Global gene expression analysis indicated that 27 pancreatic islet-specific genes were enriched in OK cells, including pancreatic progenitor markers (RBPJ, Sox9, NeuroD, and ISL1) (Fig. 2d). The enriched genes are functionally associated with $G$ protein-related cell signaling and are responsible for glucose sensing (RGS2, IL8, KCTD12, AKAP7), transcription regulation (ISL1, BEX1, CEPD, HIST2HAB, NEUROD, SOX9, RBPJ), and enzymes for pancreatic hormone processing (PCSK1, LOXL4, CPE, OAS1, PLTP, RNF128). Among the four pancreatic hormones that secret from distinct cells in the pancreatic islets, we observed upregulation of glucagon (GCG) gene (Fig. 2d), but not insulin, somatostatin, or pancreatic polypeptide (data not shown). These results suggest that human preadipocytes can be directly converted to pancreatic $\alpha$ cells by Oct 4 and Klf4 overexpression. Pancreatic cell-specific gene expression has been confirmed with real-time RT-PCR using SYBR green-conjugated gene-specific primers that show distinct expression patterns across three types of pancreatic cells $(\alpha, \beta$, and exocrine cells). ${ }^{26}$ As shown in Figure 3 a, the expression of pancreatic $\alpha$ cell markers (GCG, CNTN1, PCSK1, PDK4, RGS4, IRX2, LPPR4, LOXL2, KCTD12, KL) and $\beta$ cell markers (ISL1, HDAC9, KCNJ2) were upregulated dramatically in OK cells, while the preadipocyte marker gene (CD36, FABP4) expression decreased (Fig. 3a). The expression of pancreatic exocrine cell-specific markers (AMY1C, AMT1B, AMY1A) was highly expressed in all samples (data not shown). To assess glucagon and NeuroD protein expression (a pancreatic $\alpha$ cell product and a pancreatic progenitor marker, respectively), OK cells were seeded onto Matrigel-coated cell culture plates and cells were examined by immunocytochemistry. Our results show that all of the colonies on matrigel stained with specific antibodies against glucagon or NeuroD except for the feeder cells (Fig. 3b). In an attempt to evaluate the secretion of glucagon from the transdifferentiated pancreatic $\alpha$ cells (OK cells, Oct4 alone), the level of glucagon was measured using an ELISA from the cell culture supernatants. Although we have detected the expression of glucagon from the cell extracts, we could hardly detect the glucagon secretion. This suggests that pancreatic $\alpha$ cells are missing a component for the glucagon processing and secretion, while the cells highly express pancreatic exocrine cell markers such as AMY1C, AMT1B, and AMY1A. 

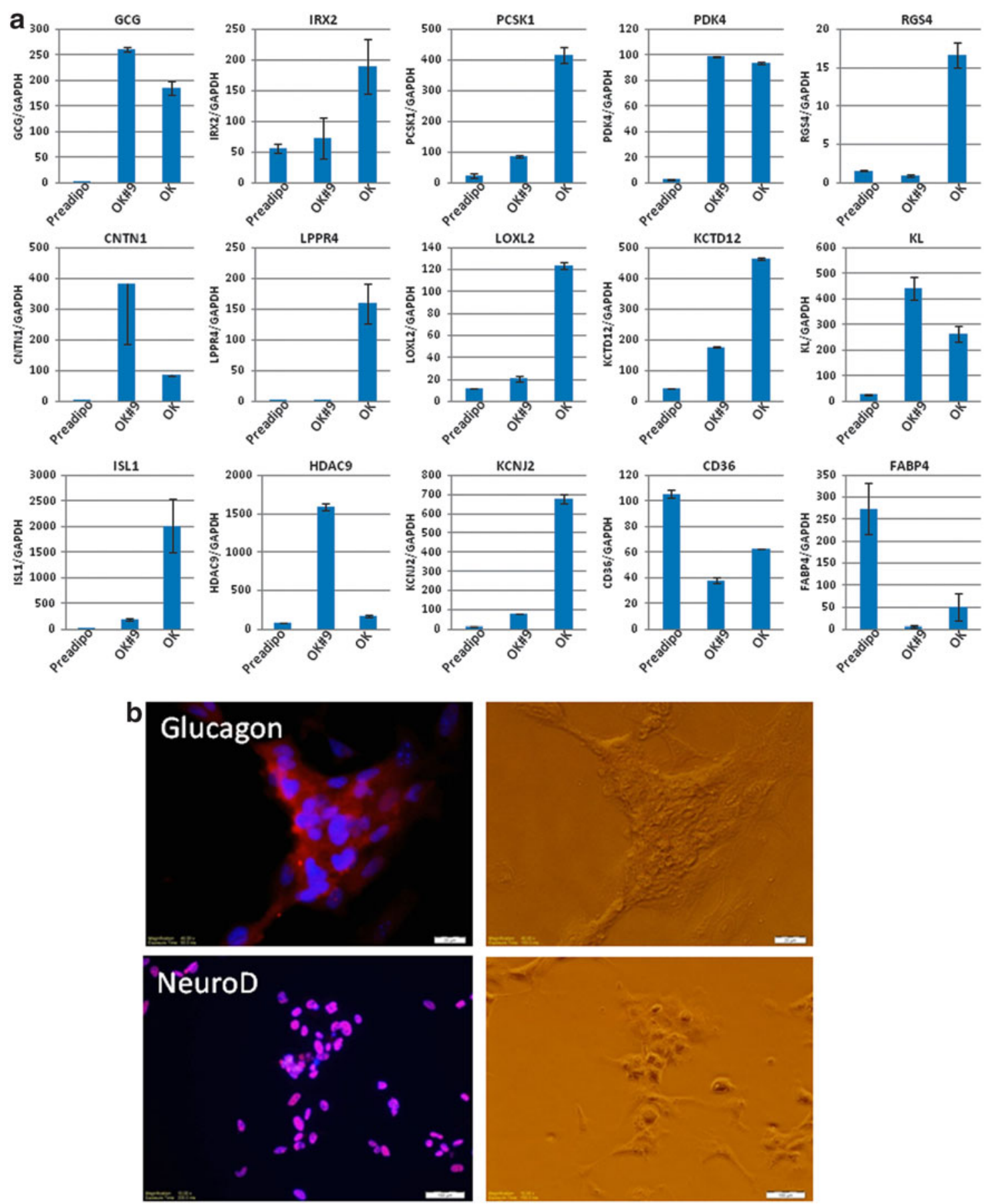

FIG. 3. Transdifferentiation of pancreatic $\alpha$ cell by Oct4 and KIf4 overexpression. (a) Real-time RT-PCR. Expression of the marker gene for pancreatic islets and adipocytes (CD36, FABP4) from two-factor-induced pancreatic $\alpha$ cell were measured by real-time RT-PCR. (b) Immunocytochemistry. The OK cells were seeded on matrigel plates and subjected to immunocytochemistry using glucagon, NeuroD-specific antibodies, and DAPI nuclear count staining. Scale bar indicates standard deviation (SD). 
C
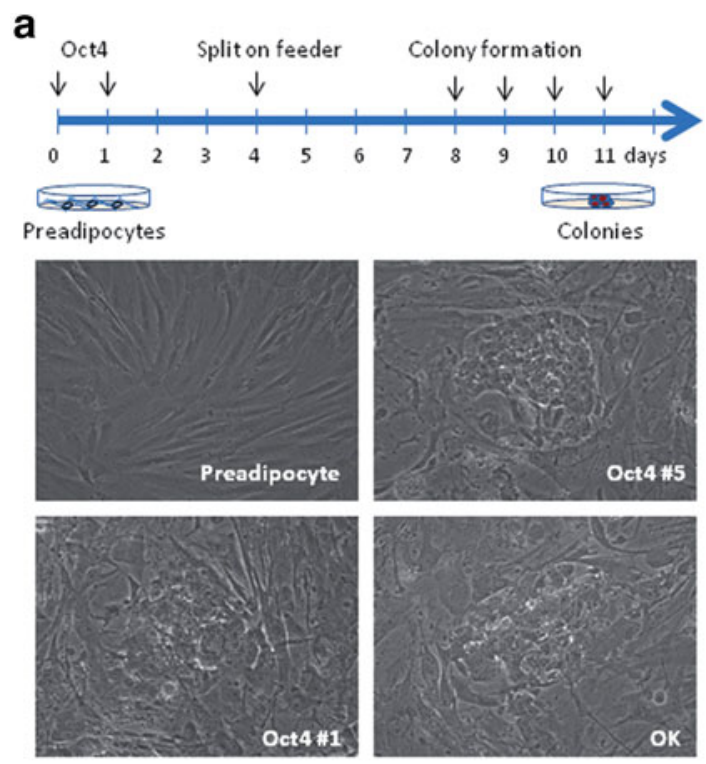
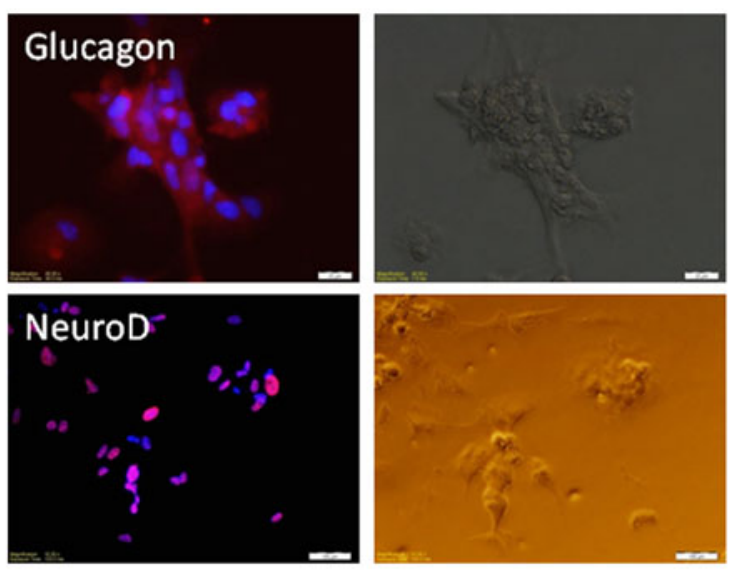

b
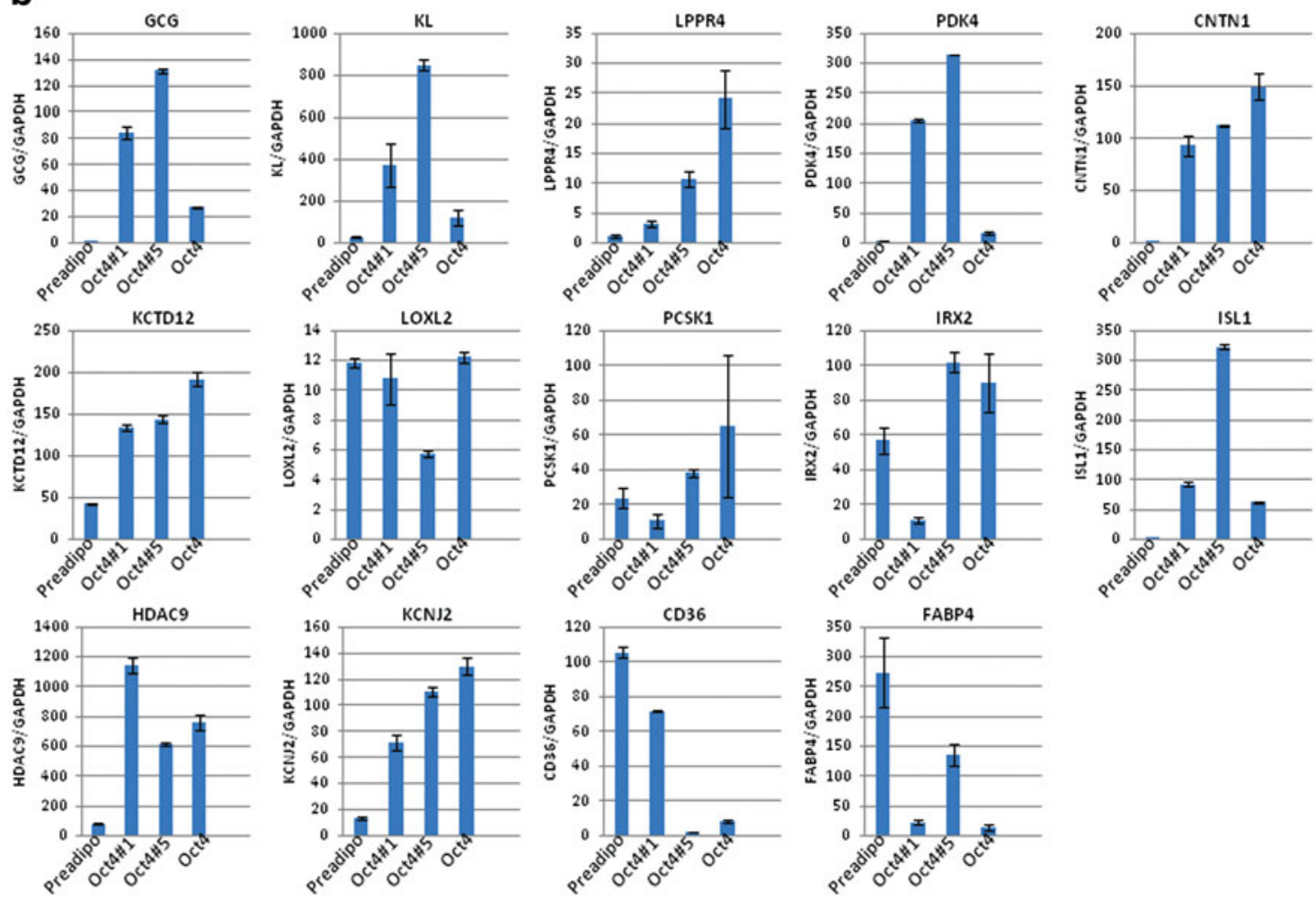

FIG. 4. Transdifferentiation of pancreatic $\alpha$ cells by Oct 4 overexpression. (a) Experimental strategy ${ }^{29}$ and micrographs of colony formation during transdifferentiation and reprogramming of human preadipocyte by lentiviral overexpression. Lentivirus that overexpresses Oct4 or two factor (Oct4, KIf4) were infected into human preadipocytes. Cells were maintained on MEF cell supplemented with the mTeSR1 medium. OK; Oct4, KIf4. (b) Real-time RT-PCR. Expression of the marker gene for pancreatic islets and adipocytes (CD36, FABP4) from Oct4induced pancreatic $\alpha$ cell (Oct4\#1 and Oct4\#5; single clones, Oct4; mixed colonies) were measured by real-time RT-PCR. (c) Immunocytochemistry. The Oct4-induced pancreatic $\alpha$ cells were seeded on matrigel plates and subjected to immunocytochemistry using glucagon, NeuroD-specific antibodies and DAPI nuclear count staining. 
Analysis of the regulatory transcription factor binding sites indicated that the promoter region of most genes that were upregulated in OK cells contain putative POU domain binding sequences ( $5^{\prime}$-ATTTGCAT-3'), but not Klf4 binding sequences, suggesting Klf4 might be dispensable for the transdifferentiation processes. To test this hypothesis, we transfected preadipocytes with Oct 4 only using a lentiviral vector and repeated the experiment using the same conditions used previously. The general outline is shown in Figure 4a. Similar to the previous two-factor overexpression, we observed colony formation at 5 days after culturing on feeder cell layer. The cells had virtually identical morphology as the two-factor (Oct4, Klf4)-induced OK cells (Fig. 4a). Next, we analyzed the pancreatic cellspecific gene and protein expression from established (Oct4\#1, Oct4\#5) and mixed colonies (Oct4). As shown in Figure $4 \mathrm{~b}$, the expression of pancreatic cell markers from Oct 4 single factor-overexpressed cells is upregulated at levels similar to two-factor (Oct4, Klf4)-induced OK cells, except for LOXL2, RGS4, and PCSK1. Glucagon and NeuroD protein expression was confirmed by immunohistochemistry (Fig. 4c). These results suggest that ectopic expression of Oct4 alone is sufficient to induce transdifferentiation of human preadipocytes into glucagon expressing pancreatic $\alpha$ cells. These results are consistent with previous findings that Oct4 and Nanog play roles in maintaining pluripotency, while Klf4 and cMyc regulate cellular division. ${ }^{27}$ This report also indicates that Klf4 is dispensable in the transdifferentiation processes. We have expanded the Oct4-induced pancreatic $\alpha$ cells into more than 10 passages without any loss of cellular proliferation properties or pancreatic marker gene expression (data not shown). Glucagon producing pancreatic $\alpha$ cells comprise $0.2-0.5 \%$ of pancreatic islets and show great plasticity to become insulin-secreting $\beta$ cells after severe $\beta$ cell loss in mouse model. ${ }^{28}$ Furthermore, Bramswig et al. have recently demonstrated that HMT inhibitor treatment facilitates the conversion of pancreatic $\alpha$ cell to insulin-secreting $\beta$ cells. ${ }^{26}$ These findings regarding the plasticity of adipose stem cells and transdifferentiation into pancreatic endocrine cells and the development of a simple and efficient method to produce pancreatic $\alpha$ cells will provide a model to advance therapeutic strategies for metabolic disease.

\section{Materials and Methods}

Cell culture and lentiviral transfection

Human preadipocytes (LaCell, LA) were maintained with DMEM supplemented with $10 \%$ FBS, $50 \mathrm{U} / \mathrm{mL}$ penicillin and $50 \mu \mathrm{g} / \mathrm{mL}$ streptomycin at $37^{\circ} \mathrm{C}$, and $5 \%$
$\mathrm{CO}_{2}$ in a humidified incubator. High-titer lentivirus, overexpressing human Oct4, Klf4, or polycistronic lentivirus, which expresses four iPS factors (STEMCCA), was purchased from System Biosciences, Cellomics, and EMD Millipore. The day before lentiviral transfection, human preadipocytes were trypsinized, counted, and seeded in six-well plates at a density of $10^{5}$ cells/ well. The next day, the culture medium was replaced with a prewarmed medium containing $5 \mu \mathrm{g} / \mathrm{mL}$ polybrene (Sigma-Aldrich) and 25 MOI of lentivirus. Lentiviral infection was repeated the next day, and the culture medium was changed with a fresh preadipocyte growth medium. When the cells reached confluence, cells were trypsinized, counted, and seeded on a prepared MEF feeder layer at a density of $5 \times 10^{4}$ cells with the mTeSR1 medium (StemCell Technology).

\section{Microarray and gene expression analysis}

Total RNA was prepared from cultures using Trizol Reagent (Life Technology) and the RNeasy Mini RNA isolation kit (Qiagen) with DNase I digestion. The RNA quality was verified using an Agilent Bioanalyzer 2100 (Agilent Technologies) using the RNA 6000 Pico Assay. Generation of double-stranded cDNA, preparation and labeling of cRNA, hybridization to HumanHT-12 v4 Expression BeadChip (Illumina), washing, and scanning were all performed according to the standard Illumina protocol. Quantitative PCR to measure mRNA expression levels was performed with PrimeTime qPCR assays (Integrated DNA Technologies) using a 7700 real-time PCR system in the genomic core facility at Pennington Biomedical Research Center. Expression levels were compared to known standard samples and were normalized to GAPDH.

\section{Immunocytochemistry}

Cells were fixed with $4 \%$ paraformaldehyde in phosphatebuffered saline (PBS) for $10 \mathrm{~min}$ and incubated for $1 \mathrm{~h}$ with antibodies specific for glucagon and NeuroD1 (Abcam). After washing thrice with PBS, cells were incubated for $1 \mathrm{~h}$ with fluorescent conjugated secondary antibody (Invitrogen). Nuclei were detected by DAPI staining (Vector shield).

\section{Acknowledgments}

The authors thank Ms. Susan Newman for technical support for microarray. We also acknowledge the use of Genomics, Cell Biology, and Imaging Core facilities that are supported, in part, by COBRE (NIH P20- 
RR021945) and NORC (NIH 1P30-DK072476) center grants from the NIH.

\section{Author Disclosure Statement}

All authors, with the exception of P.L., A.D., J.R., K.S., C.A., and J.K. acknowledge financial interests in the form of stock and/or stock options in NuPotential, Inc.

\section{References}

1. Heyworth C, Pearson S, May G, et al. Transcription factor-mediated lineage switching reveals plasticity in primary committed progenitor cells. EMBO J. 2002;21:3770-3781.

2. Xie $H$, Ye $M$, Feng $R$, et al. Stepwise reprogramming of $B$ cells into macrophages. Cell. 2004;117:663-676.

3. Kajimura S, Seale $P$, Kubota K, et al. Initiation of myoblast to brown fat switch by a PRDM16-C/EBP-beta transcriptional complex. Nature. 2009;460:1154-1158.

4. Huang $P, H e Z$, Ji $S$, et al. Induction of functional hepatocyte-like cells from mouse fibroblasts by defined factors. Nature. 2011;475:386-389.

5. Buganim Y, Itskovich E, Hu YC, et al. Direct reprogramming of fibroblasts into embryonic Sertoli-like cells by defined factors. Cell Stem Cell. 2012;11:373-386.

6. Vierbuchen T, Ostermeier A, Pang ZP, et al. Direct conversion of fibroblasts to functional neurons by defined factors. Nature. 2010;463:10351041.

7. Kim J, Su SC, Wang H, et al. Functional integration of dopaminergic neurons directly converted from mouse fibroblasts. Cell Stem Cell. 2011;9:413-419.

8. Son EY, Ichida JK, Wainger BJ, et al. Conversion of mouse and human fibroblasts into functional spinal motor neurons. Cell Stem Cell. 2011;9:205-218.

9. Yang Z, Ming GL, Song H. Postnatal neurogenesis in the human forebrain: from two migratory streams to dribbles. Cell Stem cell. 2011;9:385-386.

10. Takahashi K, Yamanaka S. Induction of pluripotent stem cells from mouse embryonic and adult fibroblast cultures by defined factors. Cell. 2006;126:663-676.

11. Kim JB, Sebastiano V, Wu G, et al. Oct4-induced pluripotency in adult neural stem cells. Cell. 2009;136:411-419.

12. Kim EB, Fang $X$, Fushan AA, et al. Genome sequencing reveals insights into physiology and longevity of the naked mole rat. Nature. 2011:479:223-227.

13. Shi Y, Do JT, Desponts $C$, et al. A combined chemical and genetic approach for the generation of induced pluripotent stem cells. Cell Stem Cell. 2008;2:525-528.

14. Tsai SY, Clavel C, Kim S, et al. Oct4 and klf4 reprogram dermal papilla cells into induced pluripotent stem cells. Stem Cells. 2010;28:221-228.

15. Liu X, Sun H, Qi J, et al. Sequential introduction of reprogramming factors reveals a time-sensitive requirement for individual factors and a sequential EMT-MET mechanism for optimal reprogramming. Nat Cell Biol. 2013;15:829-838.

16. Szabo E, Rampalli S, Risueno RM, et al. Direct conversion of human fibroblasts to multilineage blood progenitors. Nature. 2010;468:521-526.

17. Zimmerlin L, Donnenberg VS, Rubin JP, et al. Mesenchymal markers on human adipose stem/progenitor cells. Cytometry A. 2013;83:134-140.

18. Shi $Y$, Desponts $C$, Do JT, et al. Induction of pluripotent stem cells from mouse embryonic fibroblasts by Oct4 and Klf4 with small-molecule compounds. Cell Stem Cell. 2008;3:568-574.

19. Huangfu D, Maehr R, Guo W, et al. Induction of pluripotent stem cells by defined factors is greatly improved by small-molecule compounds. Nat Biotechnol. 2008;26:795-797.

20. Mikkelsen TS, Hanna J, Zhang $X$, et al. Dissecting direct reprogramming through integrative genomic analysis. Nature. 2008;454:49-55.

21. Kim J, Efe JA, Zhu S, et al. Direct reprogramming of mouse fibroblasts to neural progenitors. Proc Natl Acad Sci U S A. 2011;108:7838-7843.

22. Yang $L$, Li S, Hatch $H$, et al. In vitro trans-differentiation of adult hepatic stem cells into pancreatic endocrine hormone-producing cells. Proc Natl Acad Sci U S A. 2002;99:8078-8083.
23. Gradwohl G, Dierich A, LeMeur M, et al. neurogenin3 is required for the development of the four endocrine cell lineages of the pancreas. Proc Natl Acad Sci U S A. 2000;97:1607-1611.

24. Meivar-Levy I, Sapir T, Gefen-Halevi S, et al. Pancreatic and duodenal homeobox gene 1 induces hepatic dedifferentiation by suppressing the expression of CCAAT/enhancer-binding protein beta. Hepatology. 2007:46:898-905.

25. Kataoka K, Han SI, Shioda S, et al. MafA is a glucose-regulated and pancreatic beta-cell-specific transcriptional activator for the insulin gene. J Biol Chem. 2002;277:49903-49910.

26. Bramswig NC, Everett LJ, Schug J, et al. Epigenomic plasticity enables human pancreatic alpha to beta cell reprogramming. J Clin Invest. 2013;123:1275-1284

27. McConnell BB, Yang VW. Mammalian Kruppel-like factors in health and diseases. Physiol Rev. 2010;90:1337-1381.

28. Thorel F, Nepote V, Avril I, et al. Conversion of adult pancreatic alphacells to beta-cells after extreme beta-cell loss. Nature. 2010;464:11491154.

29. Jacobsen JP, Weikop P, Hansen $\mathrm{HH}$, et al. SK3 K+ channel-deficient mice have enhanced dopamine and serotonin release and altered emotional behaviors. Genes Brain Behav. 2008;7:836-848.

Cite this article as: Rim JS, Lewis P, Dave A, Rim J, Szuszka K, Acosta C Kim J, Kim J-D, Staszkiewicz J, Gao R, Harkins L, Eilertsen KJ (2015) Direct conversion of human preadipocytes into hematopoietic, neuronal and pancreatic $\alpha$ cells by Oct 4 and Klf4 overexpression, BioResearch Open Access 4:1, 389-397, DOI: 10.1089/biores.2015.0028.

\begin{aligned} \multicolumn{1}{c|}{ Abbreviations Used } \\ AdiPS $=$ Adipose-derived iPS cell \\ DMEM $=$ Dulbecco's Modified Eagle's Medium \\ DNMT $=$ DNA methyltransferase \\ $\mathrm{EMT}=$ epithelial-to-mesenchymal transition \\ $\mathrm{FBS}=$ fetal bovine serum \\ $\mathrm{HDAC}=$ histone deacetylase \\ $\mathrm{HMT}=$ histone methyltransferase \\ $\mathrm{ICM}=$ inner cell mass \\ $\mathrm{iPS} \mathrm{Cell}=$ induced pluripotent stem cell \\ $\mathrm{MEF}=$ mouse embryonic fibroblast \\ $\mathrm{MOI}=$ multiplicity of infection \\ $\mathrm{OK}=$ Oct4 and KIf4 \\ $\mathrm{OKSM}=$ Oct4, Klf4, Sox2, and cMyc \\ $\mathrm{PBS}=$ phosphate-buffered saline \\ $\mathrm{RT}-\mathrm{PCR}=$ reverse transcription-polymerase chain reaction \\ \end{aligned}

\section{Publish in BioResearch Open Access}

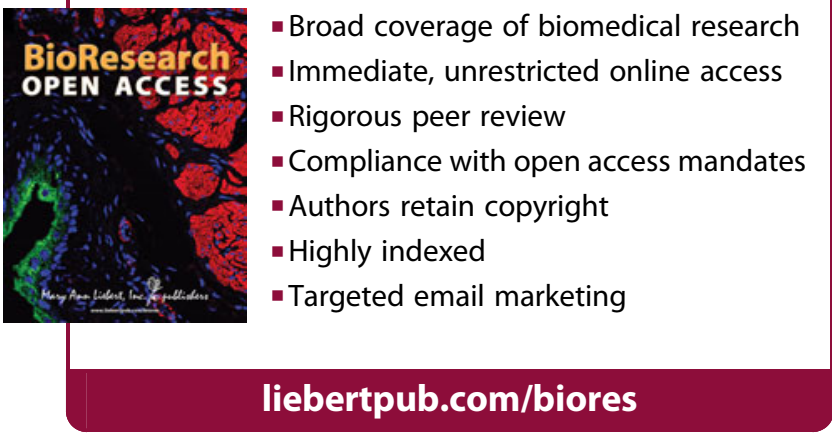

